\title{
Study on the Effect of Physical Fitness Training on Children's Cognitive Ability
}

\author{
Jianhua Deng*, Lei Ning \\ Sports Training Speciality, Yanshan University, China \\ E-mail:*245615583@qq.com \\ www.tust.edu.cn
}

\begin{abstract}
With the word physical fitness getting more and more attention in our country, physical fitness has been widely developed in our country. Therefore, in recent years, the students' physique has received attention from all aspects of society, and improving the students' physique has also become a new topic for physical education workers. I try to discuss the effect of physical fitness training on children's cognitive ability from the perspective of physical fitness training. To provide theoretical basis and reference for intervention of children's cognitive ability training methods. So as to promote the improvement of children's cognitive ability and provide guarantee. This paper studies the influence of physical fitness training on children's cognitive ability by using the methods of literature, observation and experiment. The following conclusions are drawn: 1. Before the experiment, 8 children from Qinhuangdao Aikushao Physical Fitness Center were tested for attention cognitive ability and memory cognitive ability. Among them, 8 children were deficient in cognitive ability to varying degrees. Compared before and after the experiment, physical fitness training has an effect on children's attention cognitive ability.3. Compared before and after the experiment, physical fitness training has an effect on the improvement of children's memory cognitive ability.
\end{abstract}

Keywords: Physical fitness of children; Children's cognitive ability; Experimental study

\section{Introduction}

Children are the motherland's "bud and future ". As early as 1988, at the 10th World Congress of the World Future Research Federation, experts from various countries discussed the results of the report on "the end of the children ". [1] It is generally believed that" children are the basic human resources and the creation of a world conducive to their development should be recognized as limited regional and global measures ".

However, due to the influence of the one-sided pursuit of the transition rate, the society and the school have the tendency to pay more attention to intellectual education and light sports, the students' schoolwork burden is too heavy, the rest and exercise time is seriously insufficient, and the students' interest in sports gradually weakens or even disappears ${ }^{[2]}$. The physical decline of young students seriously affects the healthy growth of children and even the future of the country and nation.

Children are in the golden development stage of life, their cognitive ability will directly affect the level of learning in the future. Therefore, according to the needs of children's own development and the requirements of improving children's basic sports ability, this study makes an experimental study on whether children's physical fitness can improve their cognitive ability ${ }^{[3]}$.The word physical fitness comes from English Physical Fitness, first appeared half a century ago by the American Association for Health, Sports, and Leisure.

After many years of development, health fitness has become an important standard to measure human physical health ${ }^{[4]}$. Scientific research and related scientific theoretical research on health fitness play an 
important role in improving human physical health and improving human living standard ${ }^{[5]}$.

Physical fitness exercises have a positive effect on the improvement of children's health and physical fitness level. These interventions aim to increase students' physical fitness level. However, most of the current studies on students' physical fitness are carried out outside the classroom, and there are few studies linking children's physical fitness with cognitive ability.

\section{Object and Methods of Study}

In this study, 7-8-year-old children in Qinhuangdao Aiku physical Fitness Center were selected to conduct physical fitness experiments. Eight 7-8-year-old children in Qinhuangdao Aiku physical Fitness Center were tested for attention cognitive ability and memory cognitive ability before the experiment. After 2 months of targeted physical fitness training, attention cognitive ability and memory cognitive ability of 8 7-8-year-old children in Qinhuangdao Aiku physical Fitness Center were tested again.

\section{Findings and analysis}

\subsection{Analysis of Children's Attention Cognitive Ability before Physical Fitness Training Intervention}

In Qinhuangdao Aiku Children's physical Fitness Center, 8 children aged 7-8 were randomly selected for the experiment. All the children participating in the experiment needed two months of targeted training to improve their cognitive ability. In the later stage of this experiment, the analysis of physical fitness training on children's attention cognitive ability and children's memory cognitive ability was analyzed. Before the experiment, in order to ensure the contrast of the experiment, according to the parents' attention ability test table and the children's memory ability test table as is shown in the Tab.1, the following table was obtained, and the attention ability and memory ability of $7-8$ years old children were analyzed.

Tab.1. Cognitive status of attention and memory

\begin{tabular}{ccccccccc} 
Name & A & B & C & D & E & F & G & H \\
\hline Attention & 18 & 12 & 10 & 7 & 13 & 10 & 15 & 14 \\
Memory & 23 & 30 & 45 & 65 & 58 & 40 & 49 & 52 \\
\hline
\end{tabular}

According to the scoring rules of children's attention ability test table, the attention cognitive level of 8 experimental children in Aiku Children's physical Fitness Center of Qinhuangdao City was obtained in Tab.2.

Tab.2. The attention cognitive ability before the experiment

\begin{tabular}{ccc}
\hline Name & Number & Percentage \\
\hline Well noted & 0 & $0 \%$ \\
mild & 4 & $50 \%$ \\
medium & 3 & $37.5 \%$ \\
severe & 1 & $12.5 \%$ \\
\hline
\end{tabular}

Through the analysis of the attention cognitive ability of 7-8-year-old children in Qinhuangdao Children's Physical Fitness Center, You know, There are 0 children with good attention skills, $0 \%$ of the total; There are four children with a slight lack of attention, 50 per cent of the total population; There are three children with moderate capacity deficits, 37.5 per cent of the total; There is one child with a severe lack of attention, $12.5 \%$ of the total number.

\subsection{Interventional training arrangements for improving children's attention awareness}

By understanding the training methods of expert physical fitness and the knowledge of physical fitness I have learned, the training contents of children's attention cognitive ability are screened out. According to the stage principle of children's physical fitness training, the training will last for 2 months

Practice is arranged in three stages of training in an advanced manner to prepare for the accuracy of the experiment. The experiment is shown in the Tab.3

Tab.3.The Content Stage of Attention Cognitive Training

\begin{tabular}{cccc}
\hline Phase & Foundation & Improvement & Strengthening \\
\hline Time & $1-3$ weeks & 4-6 weeks & 7-8 weeks \\
& Warm up & Warm up & Warm up \\
& Stretch & Stretch & Stretch \\
Training & Dumu Bridge & Porter & Canoe Bridge \\
& S Run & Color identification & S running colors \\
& Relax & Relax & Relax \\
$\begin{array}{c}\text { Number } \\
\text { of groups }\end{array}$ & 6-8 groups & 6-8 groups & 6-8 groups \\
\hline
\end{tabular}




\subsection{Analysis of Children's Attention Cognitive Ability after Physical Fitness Training Intervention}

After two months of experiments, An analysis of attention cognitive ability of 8 7-8 year old children in Qinhuangdao Aikukuo Physical Fitness Center, You know, One child with good attention skills, 12.5 per cent of the total; There are five children with a slight lack of attention, 62.5 per cent of the total; There are 2 children with moderate capacity deficits, 25 per cent of the total population; There are 0 children with severe disability, It accounts for $0 \%$ of the total as shown in Tab.4.

Tab.4. The attention cognitive ability after the experiment

\begin{tabular}{ccc}
\hline & $\begin{array}{c}\text { Number of } \\
\text { persons }\end{array}$ & Percentage \\
\hline Well & 1 & $12.50 \%$ \\
noted & 5 & $62.50 \%$ \\
mild & 2 & $25 \%$ \\
medium & 0 & $0 \%$ \\
severe & 0 \\
\hline
\end{tabular}

\subsection{Comparative Analysis of Children's Attention Cognitive Ability}

Through the comparison before and after the experiment as shown in Fig.1, 8 children with good memory ability before and after the experiment in Qinhuangdao Aikuo Children's physical ability Center had 1 person ,3 children with mild memory deficiency and 6 people after the experiment; There were 3 children with moderate memory deficiency and 1 children with severe memory deficiency. To sum up, after the experiment, the whole children's memory cognitive ability level comparison experiment came to see, has improved.

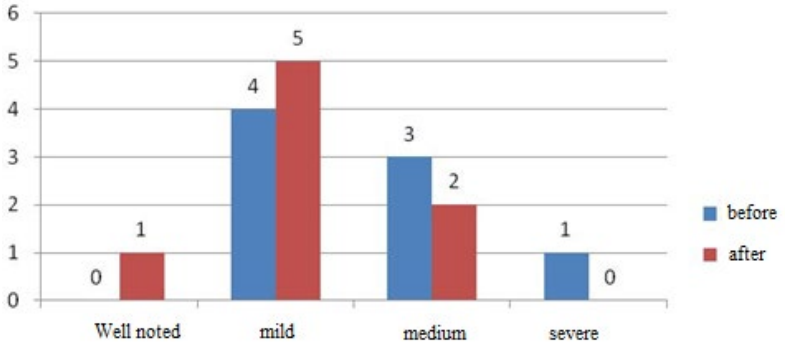

Fig. 1.Comparison of Attention Cognitive Ability of Children before and after the Experiment

\section{Conclusions}

Through the comparison of 7-8-year-old children in Qinhuangdao Aiku physical ability Center for 2 months, the physical fitness training is helpful to the improvement of children's attention cognitive ability.

\section{References}

1. Seeger JP; Thalain DH; Noor dam Physical fitness training improves physical fitness and vascular function in children with type 1 diabetes. Diabetes, Obesity \& Metabolism. 201.

2. Elbasan, Effects of chest physiotherapy and aerobic exercise training on physical fitness in young children with cystic fibrosis. Italian Journal of Pediatrics, 2012, 38 .

3. Yang Taiji .On the concept and relationship between physical fitness and physical fitness. Contemporary sports science and technology, 2018, 8(16): 178-179.

4. Zhu JUANJUAN. Research on the construction of physical education curriculum for large class children from the perspective of physical fitness. Wuhan Institute of physical education, 2018

5. Wang Zhen. Experimental research on the influence of children's physical fitness training on 3-6-year-old children's sensory integration ability. Wuhan sports College, 2018. 